\title{
Synthesis and Catalytic Activity of Supported Acenaphthoimidazolylidene Ruthenium Complex for Ring Closing Metathesis (RCM) and Ring Opening Metathesis Polymerization (ROMP)
}

\author{
Antsar R. Hlil, ${ }^{1}$ Salvador Moncho, ${ }^{1}$ Robert Tuba, ${ }^{1,2}$ Edward N. Brothers,${ }^{1}$ \\ Robert H. Grubbs, ${ }^{1,3}$ Mohammed Al-Hashimi* ${ }^{* 1}$ and Hassan S. Bazzi ${ }^{* 1}$
}

${ }^{1}$ Department of Chemistry, Texas A\&M University at Qatar, P.O. Box 23874, Doha, Qatar, E-mail: mohammed.al-hashimi@qatar.tamu; hassan.bazzi@tamu.edu

2 Institute of Materials and Environmental Chemistry, Research Centre for Natural Sciences, Hungarian Academy of Sciences Magyar tudósok körútja 2., 1519 Budapest, P.O. Box 286.

${ }^{3}$ The Arnold and Mabel Beckman Laboratories of Chemical Synthesis, Division of Chemistry and Chemical Engineering, California Institute of Technology, Pasadena, California 91125, United States.

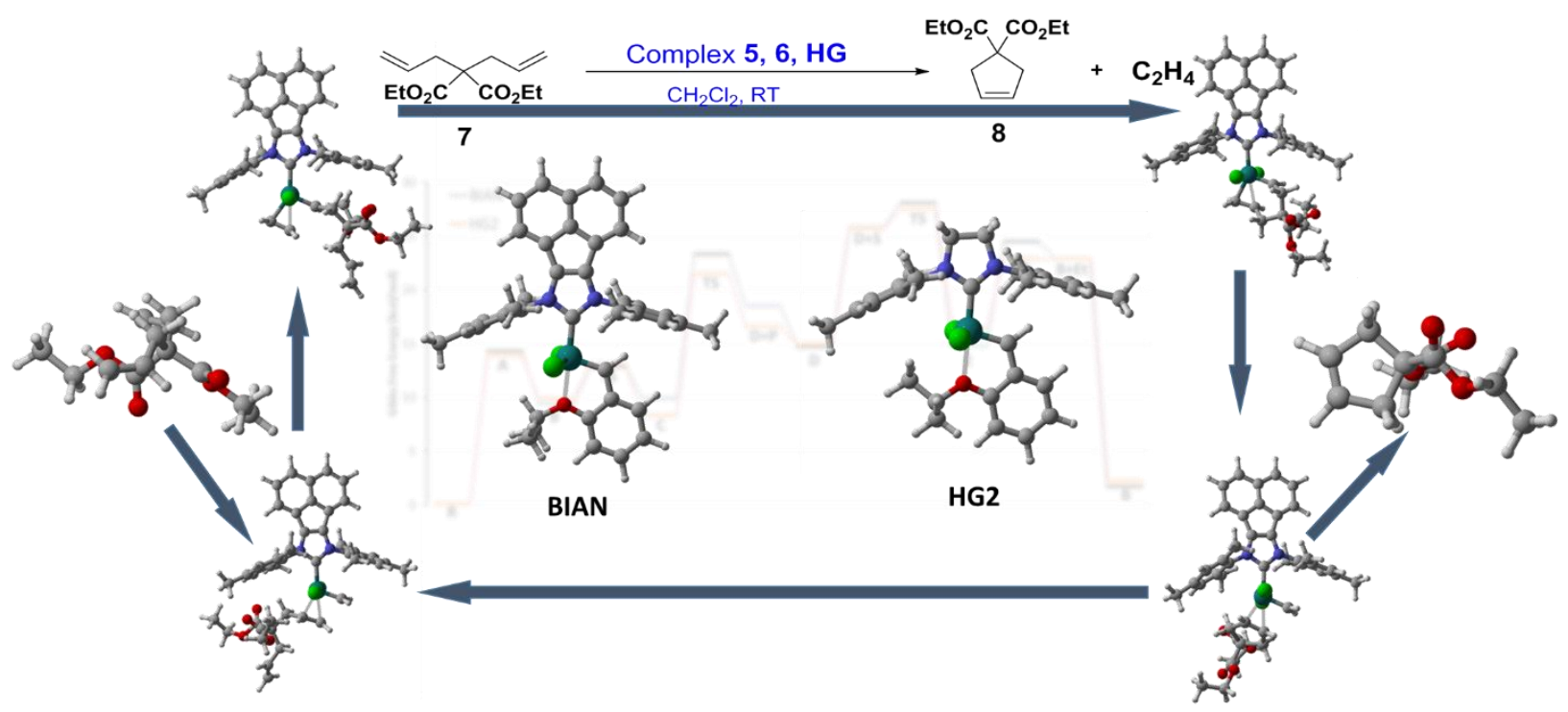

\section{Abstract}

Ruthenium catalyzed ring closing metathesis $(\mathrm{RCM})$ and ring opening metathesis polymerization (ROMP) reactions using a PIB-supported bis(arylimino)acenaphthene (BIAN) complex has been reported. The BIAN Ru-complex exhibits excellent catalytic activity and tolerates a wide range of substrates at low catalyst loadings. DFT calculations were also carried out on some selected key intermediates in the reaction mechanism to study the effect of having the conjugated aromatic moiety (BIAN ligand) in the backbone of the complex. The newly synthesized PIBBIAN Ru-complex shows comparable catalytic activities to the commercially available second generation Hoveyda-Grubbs catalyst. The recycled complex was reused for up to eight reaction cycles for the RCM of $\mathrm{N}, \mathrm{N}$-diallyl tosylamine affording high average isolated yield.

Keywords: supported ruthenium complex; ring closing metathesis; ring opening metathesis polymerization; catalysis; acenaphthenequinone 


\section{Introduction}

The progress of olefin metathesis since its discovery in the 1950s has proved to be a compelling tool in materials science, medicinal, polymer and organic chemistry. ${ }^{1-6}$ The development of highly active, stable, high functional group tolerance and easily separable ruthenium based complexes for ring closing metathesis (RCM) and ring opening metathesis polymerization (ROMP) still remains of great interest. Bis(arylimino)acenaphthene (Aryl-BIAN) ligands are reasonably inexpensive and are easily prepared via condensation reaction in acetic acid between acenaphthenequinone and an amine. ${ }^{7}$ The $\alpha$-diimine ligands have an extended $\pi$-conjugated system, making them strong $\sigma$-donors and weak $\pi$-acceptors. These collective characteristics result in the stabilization of both the low and high oxidation states of the metal center, therefore, in effect improving the catalytic activity. ${ }^{8-11}$

Transition-metal complexes of Aryl-BIAN ligands (Figure 1) have proven to be efficient homogenous catalysts for a number of organic reactions. In particular, BIAN ligands have contributed to palladium-catalyzed cross-coupling reactions to form $\mathrm{C}-\mathrm{C},{ }^{12-15}$ and $\mathrm{C}-\mathrm{N}^{16,17}$ bonds, selective hydrosilylation of carbonyl compounds using iron complexes, ${ }^{18}$ Nickel-catalyzed olefin polymerization, ${ }^{19}$ nucleophilic trifluoromethoxylation of organic halides by silver (I) complexes, ${ }^{20}$ and the catalytic hydroformylation of 1-octene using rhodium (I) complexes. ${ }^{21}$ However, the examples of ruthenium based-BIAN complexes are much fewer than other transition metal complexes (Figure 1). Drioli et al. reported the reduction of nitrobenzene by $\mathrm{CO} / \mathrm{H}_{2} \mathrm{O}$ which was catalyzed by $\mathrm{Ru}_{3}(\mathrm{CO})_{12}$ - $\mathrm{BIAN}$ based complexes. ${ }^{22}$ Lahiri and co-workers reported the electrochemical and spectroscopic characteristic associated with $\mathrm{Ru}(\mathrm{acac})_{2} \mathrm{BIAN}$ complex. ${ }^{23}$ Ragaini and co-workers have shown by using ruthenium complexes they can synthesize allylic amines from unactivated olefins. ${ }^{24}$ Two years later the same group reported the reaction between conjugated dienes and nitroarene catalyzed by Ru-BIAN complex. ${ }^{25}$

Remarkably, however, to the best of our knowledge there are no reports of ruthenium BIAN-based complexes for ROMP. To date, Merino et al. has reported the only homogenous BIAN ruthenium Hoveyda-type complexes for $\mathrm{RCM}^{26}$ The newly synthesized BIAN $\mathrm{Ru}$ complexes showed comparable catalytic activities to the unsaturated NHC Ru-complex and even higher activity was reported than the commercially available second generation Hoveyda-Grubbs catalyst. Motivated by the fact that we have not found any reported heterogeneous BIAN Hoveyda-Grubbs Rubased complexes in the open literature. Herein, we report (i) the synthesis of supported PIB based BIAN Ru-complex 5, (ii) investigate the catalytic activity of the prepared complex for both RCM and ROMP, (iii) demonstrate the strategy to reuse the catalyst in $\mathrm{RCM}$ and the ease of separating the products for the final reaction mixture. In addition, we explored the leaching of the BIAN-ruthenium catalyst, and last but not least (iv) DFT calculation was used to calculate the free energies of key intermediates and kinetic studies were used to evaluate the catalytic performance of the Ru complex in both RCM and ROMP at room. 

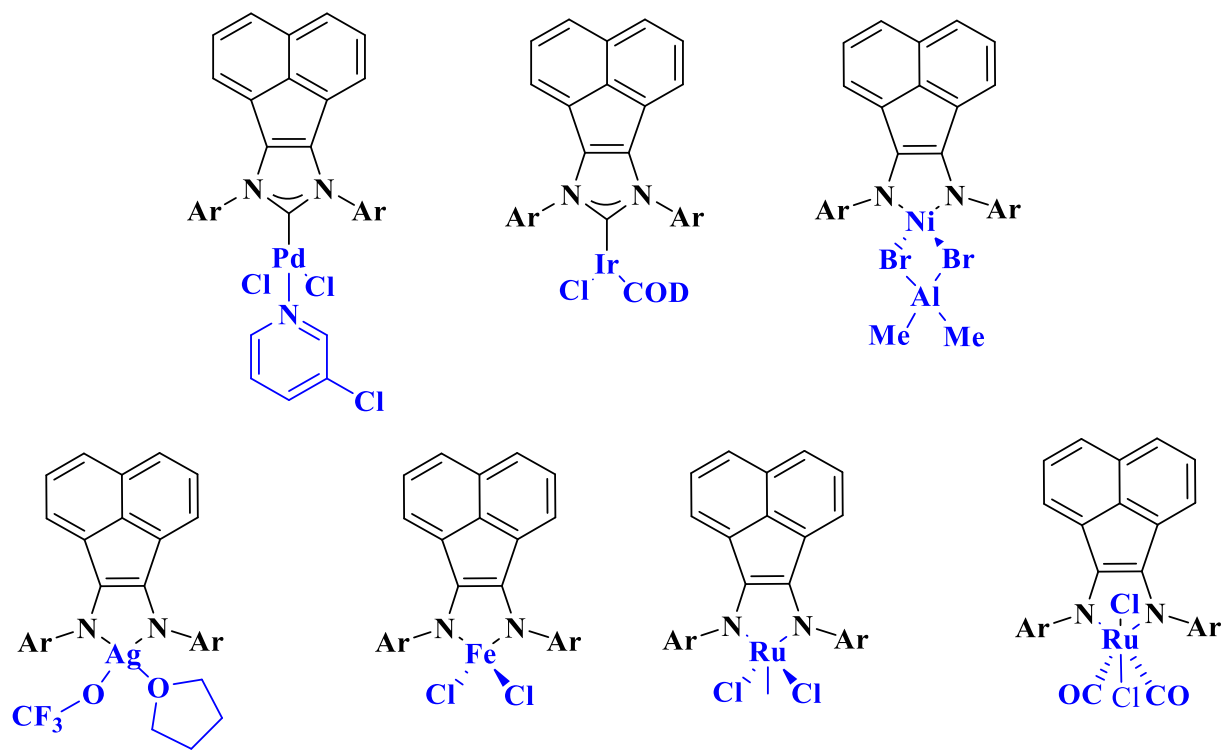

Figure 1. A selection of Aryl-BIAN transition-metal complexes.

\section{Results and Discussion}

The synthetic route to PIB BIAN Ru-complex 5 is depicted in Scheme 1. PIB-amine 1 was synthesized according to our previously reported procedure. ${ }^{27}$ Condensation reaction between the commercially available acenaphthenequinone 2 and PIB-amine 1 in acetic acid afforded PIB-diimine $3 .^{28}$ PIB-acenaphthoimidazolium salt 4 was readily accessible in a good overall yield from the corresponding PIB-diimine $\mathbf{3}$ by refluxing in ethoxy (methyl) chloride. Reaction of the imidazolium salt $\mathbf{4}$ with Hoveyda-Grubbs firstgeneration $\mathrm{Ru}$ complex in the presence of potassium tert-butoxide in a mixture of THF and toluene afforded the PIB-BIAN Ru complex 5 in $48 \%$ yield. The catalytic activity of the new Ru complex $\mathbf{5}$ was determined by conducting both RCM and ROMP reactions. 

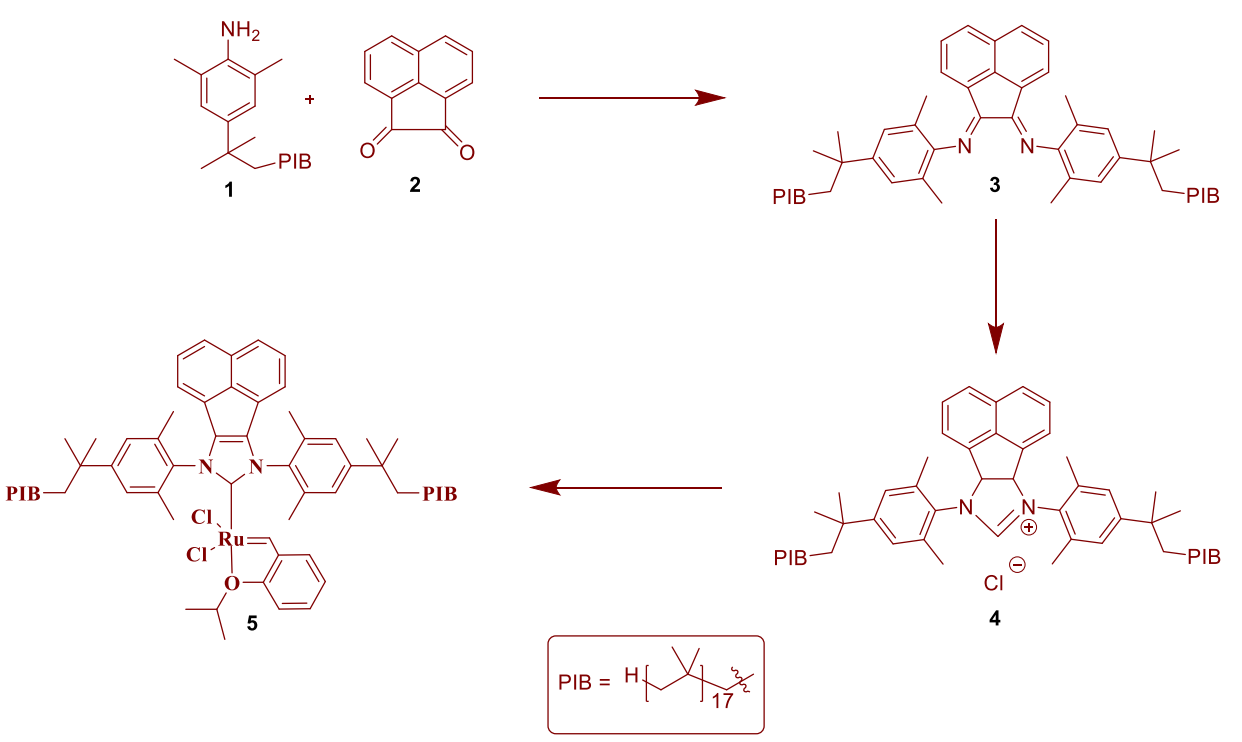

Scheme 1- Synthesis of PIB-BIAN Ru complex 5.

The catalytic activities of supported Ru-complex $\mathbf{5}$ was first investigated in the ringclosing metathesis reaction with diethyl 2, 2-diallylmalonate 7 (Figure 2, right). Moreover, a comparative study was performed between our previously reported Rucomplex $\mathbf{6},{ }^{29,30}$ commercially available HG2 and the newly supported Ru-complex $\mathbf{5}$. The reactions were carried out using $1 \mathrm{~mol} \%$ catalyst loading in $d_{2}$-dichloromethane at room temperature. The progress of the reaction over time was monitored by using ${ }^{1} \mathrm{H}$ NMR spectroscopy until full conversion of the monomer 7 was achieved. The selected kinetic data are illustrated in Figure 2. All three complexes 5,6 and HG2 afforded the desired cyclized product after $60 \mathrm{~min}$ in high yields (91 to 98\%). Interestingly, the new $\mathrm{Ru}$ complex 5 exhibited higher activity showing $56 \%$ conversion after 20 min in comparison to complex 6 which only showed 36\%. Finally, we evaluated the decomposition of the Ru complexes by running the RCM reaction over various cycles (Figure 2, left). These experiments were performed in a ${ }^{1} \mathrm{H}$ NMR tube, by placing $d_{2}$ dichloromethane in the presence of the Ru complex and the starting material. After 60 min new starting material $\left(2^{\text {nd }}\right.$ cycle) was added to the reaction mixture in the tube and the conversion of diethyl 2, 2-diallylmalonate was monitored by ${ }^{1} \mathrm{H}$ NMR spectroscopy. Interestingly, a higher conversion (94\%) was achieved after 60 min with PIB-BIAN Ru complex 5, the activity of complex HG2 was slightly lower $89 \%$. Next we left the complex in the chlorinated solvent overnight, and further tested the activity ( $3^{\text {rd }}$ cycle) in $\mathrm{RCM}$ by adding fresh substrate. After $60 \mathrm{~min}$ the experiment with PIB-BIAN Ru $\mathbf{5}$ showed slow complex decomposition during the RCM reaction, the catalytic activity was measured to be $76 \%$ after $60 \mathrm{~min}$, upon prolonged reaction times (120 $\mathrm{min}$ ) 83\% conversion to the product was observed. On the other hand, complex HG2 showed no sign of catalytic activity, which is appointed to the decomposition of the active Rucarbene species. Presumably the better catalytic activity and longer complex life time of 5 in comparison to complex HG2 is expected to be as a result of the steric hindrance associated with the PIB on the aryl rings. 

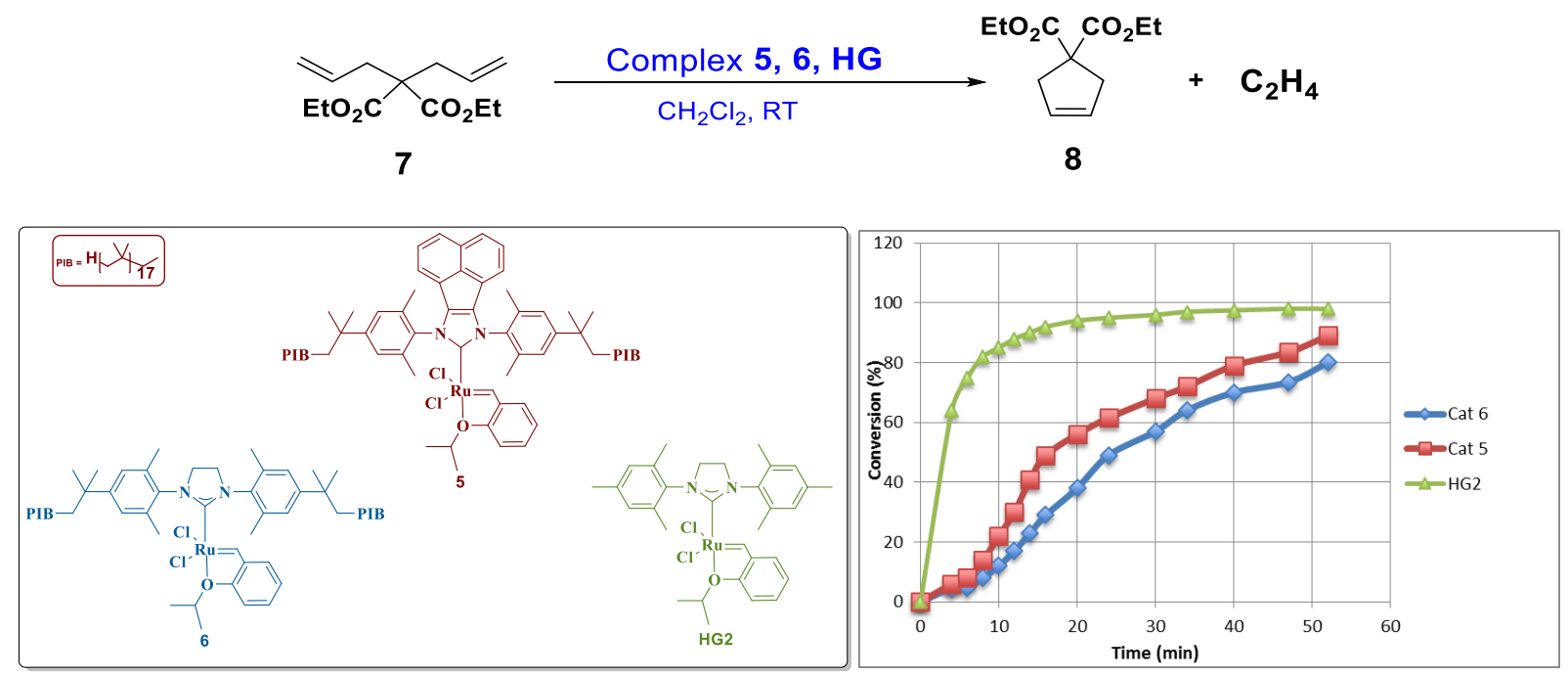

Figure 2- RCM of diethyl 2, 2-diallylmalonate 7 (right) and the RCM conversion of monomer 7 (left) using PIB-BIAN Ru complex 5, 6 and HG2.

\section{DFT calculations}

To the best of our knowledge, there has been no reported computational analysis on a BIAN-Ru Hoveyda-Grubbs complex, although the value of theory in understanding metal complexes has been well documented.' Density functional theory (DFT) calculations were carried out on some key intermediates in the RCM reaction mechanism to study the effect of having a conjugated aromatic moiety (i.e., the BIAN ligand) in the backbone of the complex, as depicted in Scheme 2. In particular, the reaction pathway with diethyl 2, 2-diallylmalonate 7 as a substrate was computationally studied, contrasting the route with the Ru-BIAN complex 5 and the HG2 complex. Please note that our model compound did not include the PIB fragment, due the flexible hydrocarbon chains, since the sampling of all potential conformers is not practical. The optimized geometry of the Ru-BIAN complex precursor $\mathbf{R}$ was compared to the literature $\mathrm{X}$-ray structure." There was obvious agreement between theory and experiment for the Ru-Ligand distances. 


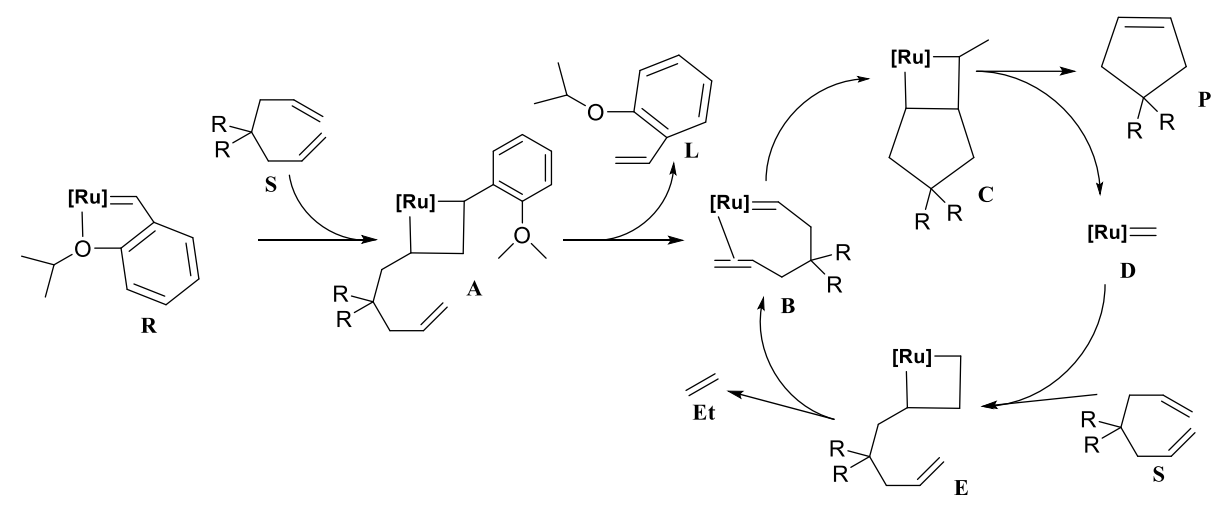

Scheme 2- Simplified reaction mechanism of the RCM.

We have selected a few key intermediates based on previous studies of the reaction mechanism. iii-iv Herein, two different parts of the mechanism are considered (scheme 2): (a) the conversion of the pre-catalyst to the active species and (b) the catalytic cycle itself. It is well-established that the metathesis reaction in the Grubb's family of catalysts occurs through the formation of 4-membered rings, built from the metal, one alkylidene carbon and an olefin. For this reason, our work focused on these intermediates, and the transition states corresponding to the formation and breaking of this ring. To model the initiation phase, we evaluated the relative stability of the 4-membered ring intermediates A. For the catalytic cycle, we considered the relative stabilities of two different intermediates containing 4-atom rings, one corresponding to the ring closing step $\mathbf{C}$ and the other to the release of ethylene $\mathbf{E}$. The free energy profile with relative Gibbs free energies for the intermediates and their transition states are shown in Figure 3. In general, the energy pathways for the two complexes are very similar, and the presence of the aromatic backbone is not expected to alter reactivity. There is a remarkable agreement with the experimental results, as both complexes are found to be highly active RCM catalysts. The small differences in the energetic pathways suggest different behaviors for the two different parts of the reaction. The initiation step was calculated to be slower in the BIAN Ru-complex 5, thus intermediate $\mathbf{A}$ is slightly less stable when using this complex. However, once the active catalyst is formed, the RCM reaction is slightly faster for the BIAN Ru-complex $\mathbf{5}$, because the relative energies are lower in the catalytic cycle. Calculations showed that the free energy difference between the least stable transition state (TSD-E) and the most stable intermediates $\mathbf{C}$ or $\mathbf{B}$ for the BIAN Ru-complex 5 was $18.7 \mathrm{kcal} \mathrm{mol}^{-1}$ and $19.2 \mathrm{kcal} \mathrm{mol}^{-1}$ for the $\mathrm{HG} 2$ complex. A relatively small difference of $0.5 \mathrm{kcal} \mathrm{mol}^{-1}$ between the commercially available complex and the synthesized BIAN Ru-complex 5 was observed. 


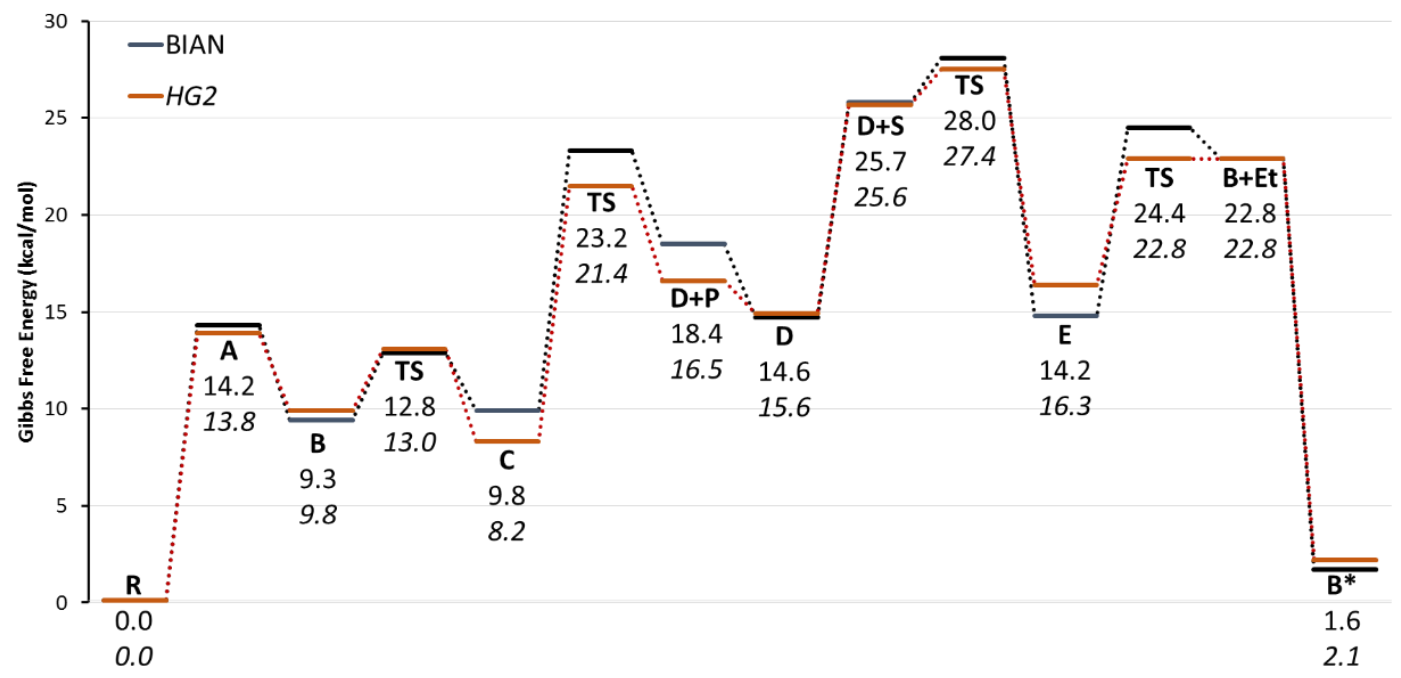

Figure 3- DFT free energy profile of the catalytic cycle. $\mathbf{B}^{*}$ represents the energy level of the intermediate $\mathbf{B}$ at the end of the cycle.

Optimized geometries for selected species are shown in Figure 4 (see supporting information for a more complete set of geometries, including those without the BIAN moiety). Despite having the bulkier, conjugated BIAN ligand, there is no significant geometry change in the coordination sphere of the metal. For example, in intermediate C the distances between the metal and the coordinated atoms change minimally (less than $0.012 \AA$ ). For $\mathbf{D}$, the values are very similar, with a maximum deviation of $0.013 \AA$ for the Ru-BIAN distance. It must be noted that, despite the lack of aromaticity in the HG2 ligand, the geometry is very similar to the Ru-BIAN complex, because the delocalized density around the nitrogen-carbene-nitrogen moiety enforces planarity of the ring, with dihedral angles (N-C-C-N) below 11.1 degrees in the calculated intermediates.

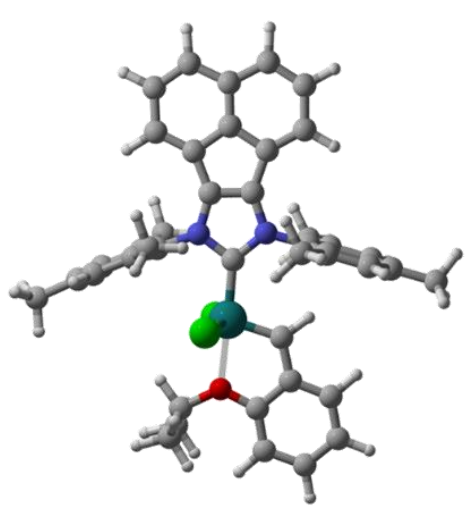

$\mathbf{P}$

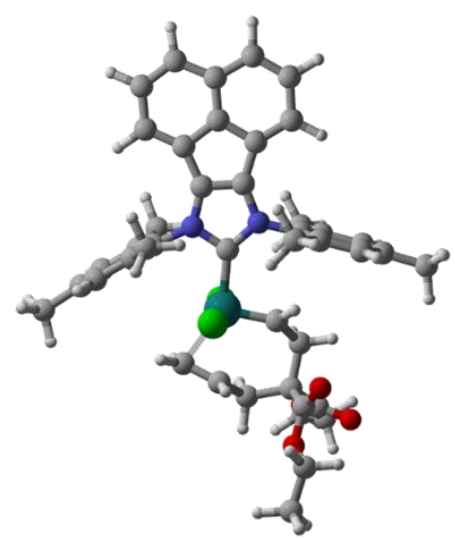

B

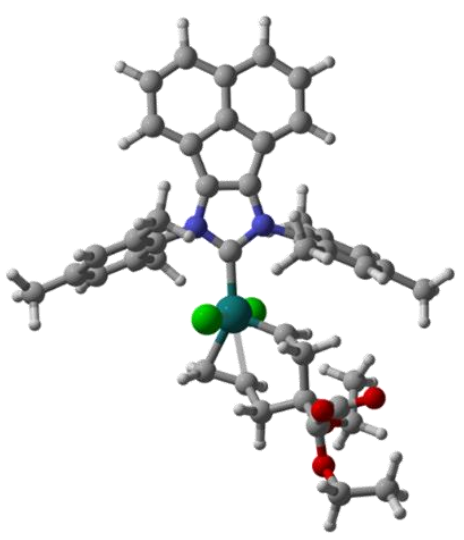

$\mathrm{TS}_{\mathrm{B}-\mathrm{C}}$ 


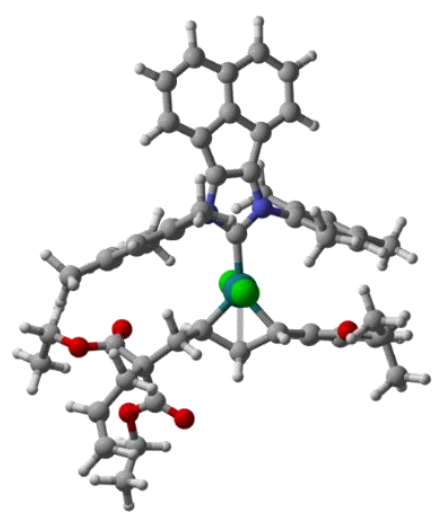

A

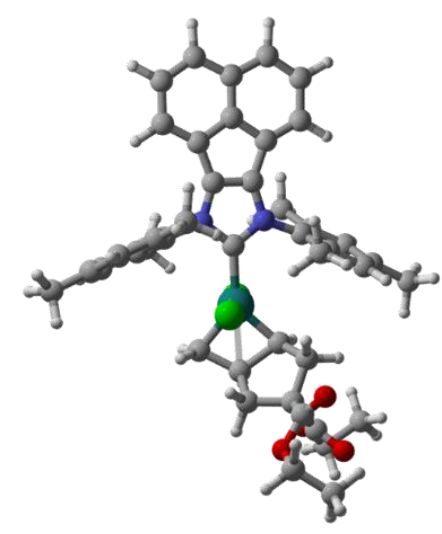

C

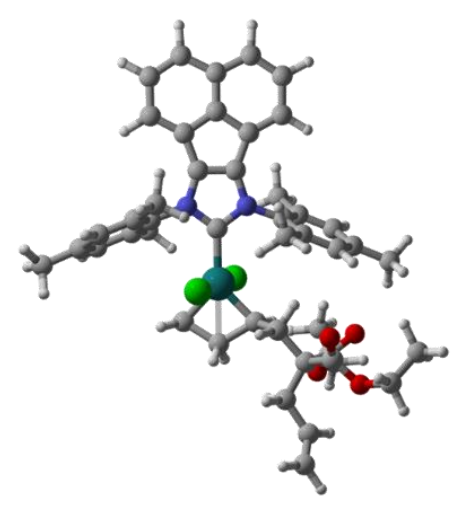

E

Figure 4- DFT optimized geometries of BIAN Ru-complexes 5 (excluding the PIB ligand for clarity).

Inspired by these promising results obtained for the kinetic studies, we decided to investigate the performance of the new supported Ru-complex 5 for RCM on a wider range of dienes as depicted in Table 2 . Using $1 \mathrm{~mol} \%$ of complex 5 (comparatively a very low loading in comparison to the $5 \mathrm{~mol} \%$ and the high temperatures that are used in the literature), ${ }^{31}$ efficient conversion to the cyclized dienes (Table 1, entries 1-6) was achieved within 60 min (70-99\%). Even the slightly hindered diene 11 (Table 1, entry 5) surprisingly formed the desired cyclic product in $95 \%$ after 60 min. However, cyclization of tosylamine 12 to the six membered product was slightly more cumbersome, resulting in $70 \%$ conversion after $60 \mathrm{~min}$. Nevertheless, upon prolonged reaction time (4h) $97 \%$ conversion was achieved, respectively (Table 1, entry 6). Notably, the supported Complex 5 displayed remarkable recyclability, without a decrease in catalytic activity. Accordingly, recycling experiments for monomers 7-8 and 12 (Table 1, entries 1, 2 and 6 ) were conducted after each run, by carrying out the reaction in heptane and then extracting the product in acetonitrile. Then a fresh monomer was added to the heptane layer and reused. In the case of the tosyl amine monomers 9-11 (Table 1, entries 3-5) the cyclic products precipitated from solution, therefore the recycling was made easy, by just adding fresh monomers to the hexane solution that contained the soluble complex 5. The recycled complex was reused for up to eight reaction cycles for the RCM of $N, N$ diallyl tosylamine 9 (Table 1, entry 3, 92\% conversion) affording an average isolated yield of $90 \%$.

Table 2- RCM of dienes using complex 5, 6 and HG.

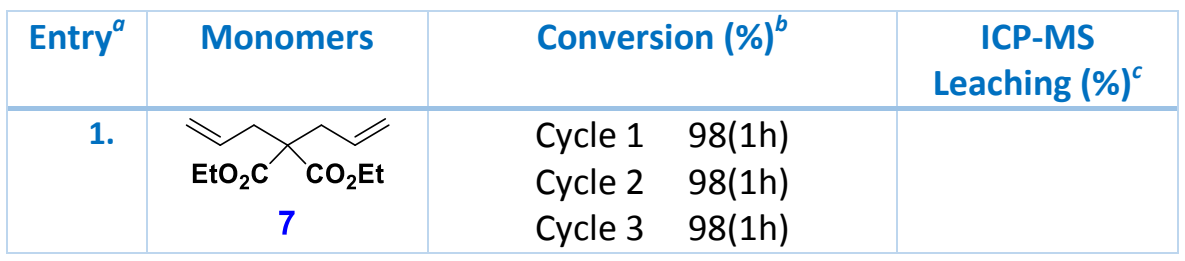




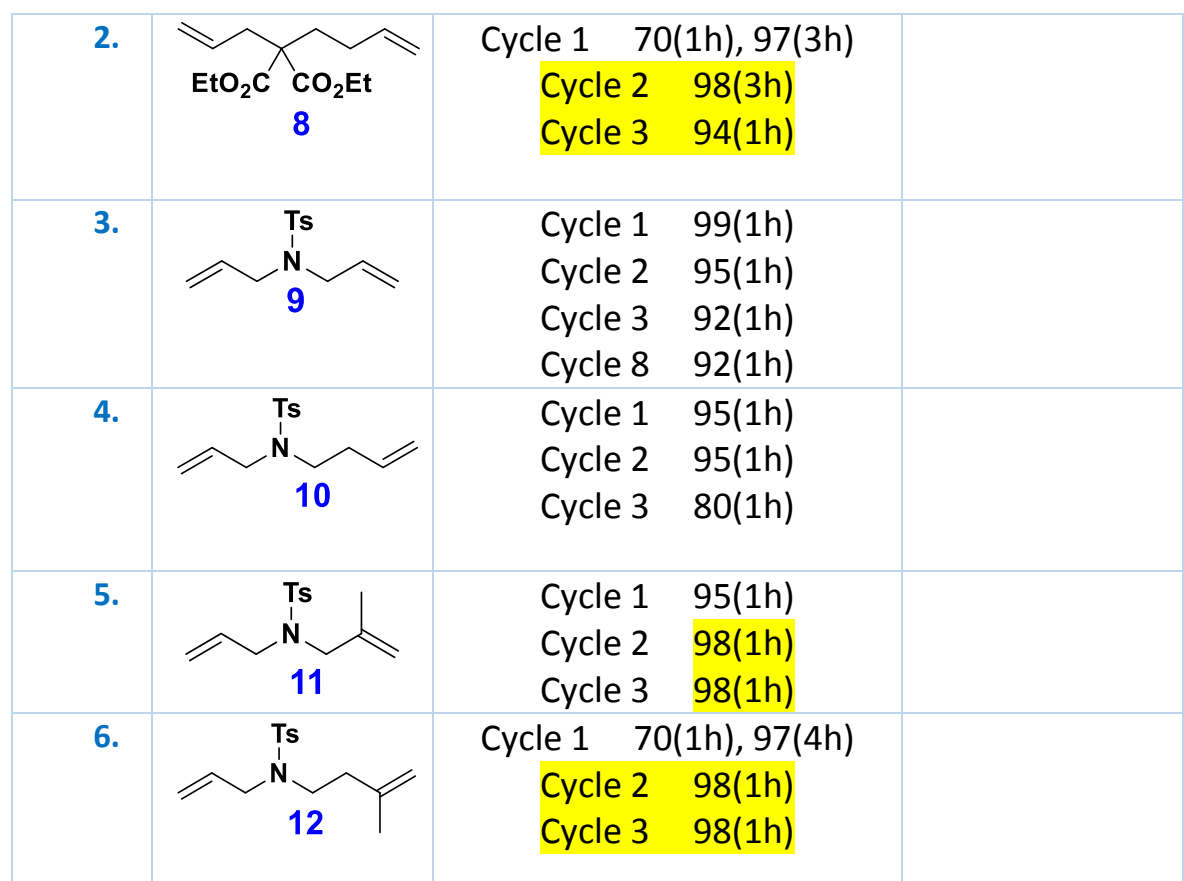

a 1 mol\% catalyst loading in $\mathrm{CH}_{2} \mathrm{Cl}_{2}(1 \mathrm{~mL})$ at $\mathrm{rt}$; ${ }^{\mathrm{b}}$ Conversion yield determined by ${ }^{1} \mathrm{H}$ NMR spectroscopy after $60 \mathrm{~min} ;{ }^{\mathrm{C}}$ Measurements were done using ICP-MS analysis, the $\mathrm{mol} \% \mathrm{Ru}$ is the \% leached into the polymer; ${ }^{d}$

To examine the scope of complex 5 we next carried out the ROMP of variously prepared norbornene derivatives 12-27 (Table 3). The ROMP of the monomers can be performed efficiently at room temperature in $60 \mathrm{~min}$ to afford the desired homopolymers in good yields. The polymers were fully characterized using ${ }^{1} \mathrm{H}$ NMR spectroscopy, the molecular weights and polydispersities were determined using GPC (Table 3).

Table 3- ROMP of norbornene derivatives using complex 5.

\begin{tabular}{|c|c|c|c|c|}
\hline Entry $^{a}$ & Monomer & $\begin{array}{c}M_{w}, M_{n}, \\
\mathrm{PDI}^{\mathrm{b}}\end{array}$ & Yield $\%^{\mathrm{c}}$ & $\begin{array}{l}\text { ICPMS- }^{d} \\
\text { leaching\% }\end{array}$ \\
\hline 1. & & Insoluble & 70 & \\
\hline 2. & & Oligomers & 15 & \\
\hline 3. & & $\begin{array}{l}50 \% \\
\text { conversion }\end{array}$ & 10 & \\
\hline
\end{tabular}




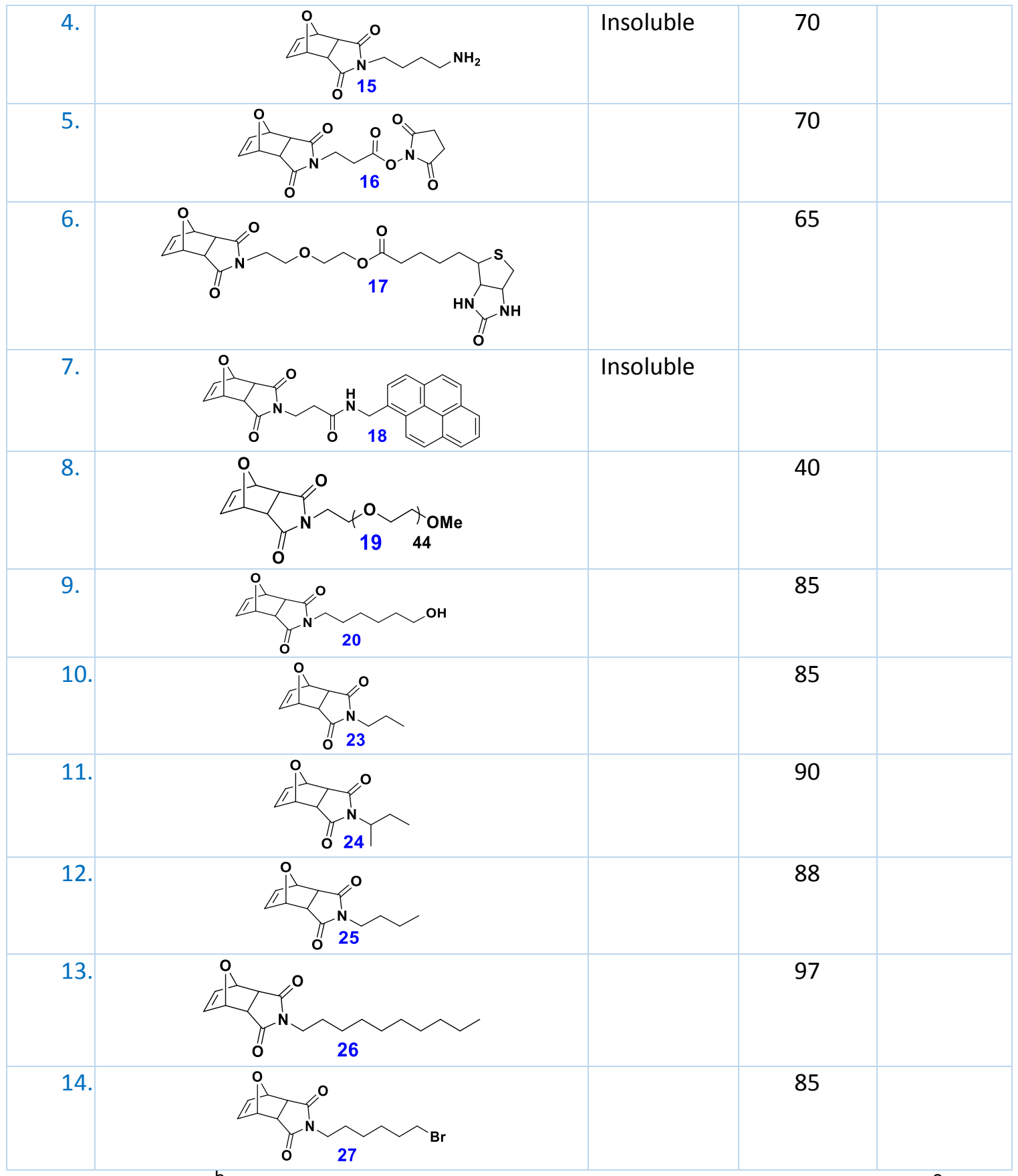

a 1 mol\% catalyst; ${ }^{b}$ Molecular weight and PDI were determined by GPC; ${ }^{c}$ Isolated yield; ${ }^{d}$ Measurements were done using ICP-MS analysis, the mol $\% \mathrm{Ru}$ is the $\%$ leached into the polymer.

\section{Conclusions}

In conclusion, we have reported on the synthesis of a supported Ru-BIAN complex $\mathbf{5}$ that can be used efficiently both for RCM and ROMP at very low catalyst loading (1 mol\%), to afford the desired products in good yields. In addition, the Ru-complex was 
found to be active and demonstrated no significant loss of catalytic activity after repeated use in RCM. The products were efficiently and easily purified form the Ruresidues, indicating low levels of ruthenium leaching into the final product, in comparison to the commercially available Ru-complex. DFT calculation were carried out to offer an introspective understanding of the relationship between the catalytic activity and the conjugated aromatic moiety (BIAN ligand) in the backbone of the Ru-complex.

\section{Experimental Section}

\section{Materials and Reagents}

PIB Ru-catalyst 6 was prepared according to literature procedure. PIB (Glissopal 1000) was a gift from BASF. All other reagents were purchased from Sigma Aldrich. All reactions were carried out under argon using solvents and reagents as commercially supplied without further purification, unless otherwise stated. ${ }^{1} \mathrm{H} N M R$ and ${ }^{13} \mathrm{C}$ NMR spectra were recorded on a Bruker Avance II 400 spectrometer, using the residual solvent resonance of THF or tetramethylsilane (TMS) as an internal reference and are given in ppm. Number-average $\left(M_{n}\right)$ and weight average $\left(M_{w}\right)$ were determined by GPC analyses, which were carried out using a Viscotek GPC Max VE 2001 instrument with a Viscotek TDA 302 triple array detector and Viscotek Org Guard column with three (in series) mixed medium columns $(\mathrm{LT} 5000 \mathrm{~L})$ at $35^{\circ} \mathrm{C}$ with a flow rate of $1.0 \mathrm{~mL} / \mathrm{min}$. A 12 point universal calibration curve was recorded using narrow polydispersity polystyrene standards. Flash chromatography (FC) was performed on silica gel (Merck Kieselgel 60 F254 230-400 mesh) unless otherwise indicated. Thin-layer chromatography was performed on Merck aluminum-backed plates precoated with silica $(0.2 \mathrm{~mm}, 60 \mathrm{~F} 254)$. ICP-MS data were obtained using a Perkin-Elmer ELAN DRCe instrument.

\section{Computational Details}

All DFT calculations were performed with the development version of the Gaussian suite of programs. ${ }^{v}$ Geometries were optimized using the M06L ${ }^{\text {vi }}$ density functional, with the double- $\zeta$ basis set def2-SZVP. vii Energies were refined with a single-point calculation using a larger basis set (the triple- $\zeta$ basis set def2-TZVPP) on the optimized geometry. Both basis sets substituted the core electrons of the ruthenium by an effective core potential. The accuracy of using the smaller basis set for optimization was by fully optimizing the geometry of a select subset if complexes with the largest basis set. This had little effect, as deviations in the relative energies where below 0.2 $\mathrm{kcal} / \mathrm{mol}$. To ensure numerical accuracy, integration grids larger than the default are used for geometry optimization ('ultrafine' grid with 99 radial shells of 590 points) and for the final energy ('superfine' grid 150 or 225 shells of 974 points). The reported geometries were confirmed as minima or transition states according to their number of imaginary frequencies. Except stated otherwise, all the energies reported in this paper are Gibbs energies computed at $298.15 \mathrm{~K}$ and $1 \mathrm{~atm}$, and are expressed in $\mathrm{kcal} / \mathrm{mol}$. Vibrational terms were calculated at the same level of theory as the geometry 
optimization. Figures of molecular geometries included in this work were rendered using CYLview. viii

\section{General procedure for the ring-closing metathesis}

A mixture of the PIB-supported Ru complex 5 (1 mol\%, $0.002 \mathrm{mmol}, 5.7 \mathrm{mg}$ ) and $\mathrm{N}, \mathrm{N}$ diallyl tosylamine $9(50 \mathrm{mg}, 0.2 \mathrm{mmol})$ were dissolved in heptane $(1 \mathrm{~mL})$. The reaction mixture was stirred for $60 \mathrm{~min}$ at room temperature. As the reaction proceeded, the product 1-tosyl-2, 5-dihydro-1H-pyrrole P9 precipitated out of the solution and was separated from the reusable heptane solution of the catalyst. The product was dried under reduced pressure and the heptane layer was directly recycled in subsequent reactions.

\section{General procedure for the ring-opening metathesis polymerization}

In a glove box, the appropriate monomer $\mathbf{M} 12(30 \mathrm{mg}, 0.1 \mathrm{mmol})$ and the PIB-supported Ru complex $5\left(1 \mathrm{~mol} \%, 2.85 \mathrm{mg}, 1 \times 10^{-3} \mathrm{mmol}\right)$ were weighed into a Schlenk flask and dissolved in anhydrous DCM $(1 \mathrm{~mL})$, and the reaction was stirred for $60 \mathrm{~min}$ at room temperature. The polymerization was terminated by injecting $0.1 \mathrm{~mL}$ of ethyl vinyl ether and the crude mixture was analyzed be ${ }^{1} \mathrm{H}$ NMR spectroscopy to determine the \% monomer conversion.

Separation of PIB Ru-catalyst from the product

The polymer DCM solution was evaporated to $0.5 \mathrm{~mL}$ and the crude polymer was precipitated in hexane $(1 \mathrm{~mL})$. The hexane layer contained the supported Ru-complex and the polymer precipitated out as off white solid.

\section{Acknowledgements}

The authors gratefully acknowledge support of this work from the Qatar National Research Fund project number: NPRP X-095-1-024. 


\section{References}

(1) Van de Weghe, P.; Eustache, J.; Cossy, J. Metathesis reactions. General considerations. Curr Top Med Chem 2005, 5, 1461-1472.

(2) Clavier, H.; Grela, K.; Kirschning, A.; Mauduit, M.; Nalon, S. P. Sustainable concepts in olefin metathesis. Angew Chem Int Edit 2007, 46, 6786-6801.

(3) Monsaert, S.; Vila, A. L.; Drozdzak, R.; Van Der Voort, P.; Verpoort, F. Latent olefin metathesis catalysts. Chem Soc Rev 2009, 38, 3360-3372.

(4) Grubbs, R. H. Olefin metathesis. Tetrahedron 2004, 60, 7117-7140.

(5) Hoveyda, A. H.; Zhugralin, A. R. The remarkable metal-catalysed olefin metathesis reaction. Nature 2007, 450, 243-251.

(6) Hopkinson, M. N.; Richter, C.; Schedler, M.; Glorius, F. An overview of N-heterocyclic carbenes. Nature 2014, 510, 485-496.

(7) Hill, N. J.; Vargas-Baca, I.; Cowley, A. H. Recent developments in the coordination chemistry of bis(imino)acenaphthene (BIAN) ligands with s- and p-block elements. Dalton T 2009, 240253.

(8) Fedushkin, I. L.; Khvoinova, N. M.; Skatova, A. A.; Fukin, G. K. Oxidative addition of phenylacetylene through $\mathrm{C}-\mathrm{H}$ bond cleavage to form the Mg-II-dpp-bian complex: Molecular structure of $[\mathrm{Mg}\{\mathrm{dpp}-\mathrm{bian}(\mathrm{H})\}(\mathrm{C} \mathrm{CPh})(\mathrm{thf})(2)]$ and its diphenylketone insertion product $[\mathrm{Mg}$ (dpp-bian)(center dot)\{OC(Ph-2)C CPh\}(thf)]. Angew Chem Int Edit 2003, 42, 5223-5226.

(9) Marion, N.; Diez-Gonzalez, S.; Nolan, S. P. N-heterocyclic carbenes as organocatalysts. Angew Chem Int Edit 2007, 46, 2988-3000.

(10) Tu, T.; Malineni, J.; Dotz, K. H. A novel pyridine-bridged bis-benzimidazolylidene pincer palladium complex: Synthesis and catalytic properties. Adv Synth Catal 2008, 350, 1791-1795. 
(11) Reeske, G.; Hoberg, C. R.; Hill, N. J.; Cowley, A. H. Capture of phosphorus(I) and arsenic(I) moieties by a 1,2-bis(arylimino)acenaphthene (aryl-BIAN) ligand. A case of intramolecular charge transfer (vol 128, pg 2800, 2006). J Am Chem Soc 2006, 128, 5300-5300.

(12) Liu, Z. L.; Dong, N. N.; Xu, M. Z.; Sun, Z. M.; Tu, T. Mild Negishi Cross-Coupling Reactions Catalyzed by Acenaphthoimidazolylidene Palladium Complexes at Low Catalyst Loadings. J Org Chem 2013, 78, 7436-7444.

(13) Ban, Q.; Zhang, J.; Liang, T. L.; Redshaw, C.; Sun, W. H. 2,6-Dibenzhydryl-N-(2aryliminoacenaphthylenylidene)-4-chlorobenzenaminopalladium dichlorides: Synthesis, characterization, and use as catalysts in the Heck-reaction. J Organomet Chem 2012, 713, 151-156.

(14) Grasa, G. A.; Singh, R.; Stevens, E. D.; Nolan, S. P. Catalytic activity of Pd(II) and Pd(II)/DAB-R systems for the Heck arylation of olefins. J Organomet Chem 2003, 687, 269-279.

(15) Grasa, G. A.; Hillier, A. C.; Nolan, S. P. Convenient and efficient Suzuki-Miyaura crosscoupling catalyzed by a palladium/diazabutadiene system. Org Lett 2001, 3, 1077-1080.

(16) Tu, T.; Fang, W. W.; Jiang, J. A highly efficient precatalyst for amination of aryl chlorides: synthesis, structure and application of a robust acenaphthoimidazolylidene palladium complex. Chem Commun 2011, 47, 12358-12360.

(17) Dastgir, S.; Coleman, K. S.; Cowley, A. R.; Green, M. L. H. Synthesis, Structure, and Temperature-Dependent Dynamics of Neutral Palladium Allyl Complexes of Annulated Diaminocarbenes and Their Catalytic Application for C-C and C-N Bond Formation Reactions. Organometallics 2010, 29, 4858-4870.

(18) Wekesa, F. S.; Arias-Ugarte, R.; Kong, L.; Sumner, Z.; McGovern, G. P.; Findlater, M. IronCatalyzed Hydrosilylation of Aldehydes and Ketones under Solvent-Free Conditions. Organometallics 2015, 34, 5051-5056.

(19) Gao, W.; Xin, L.; Hao, Z. Q.; Li, G. D.; Su, J. H.; Zhou, L. J.; Mu, Y. The ligand redox behavior and role in 1,2-bis[(2,6-diisopropylphenyl)imino]-acenaphthene nickel-TMA(MAO) systems for ethylene polymerization. Chem Commun 2015, 51, 7004-7007.

(20) Chen, S. X.; Huang, Y. J.; Fang, X.; Li, H. H.; Zhang, Z. X.; Hor, T. S. A.; Weng, Z. Q. ArylBIAN-ligated silver(I) trifluoromethoxide complex. Dalton T 2015, 44, 19682-19686.

(21) Dastgir, S.; Coleman, K. S.; Cowley, A. R.; Green, M. L. H. Stable crystalline annulated diaminocarbenes: coordination with rhodium(I), iridium(I) and catalytic hydroformylation studies. Dalton T 2009, 7203-7214.

(22) Vigano, M.; Ragaini, F.; Buonomenna, M. G.; Lariccia, R.; Caselli, A.; Gallo, E.; Cenini, S.; Jansen, J. C.; Drioli, E. Catalytic Polymer Membranes under Forcing Conditions: Reduction of Nitrobenzene by $\mathrm{CO} / \mathrm{H} 2 \mathrm{O}$ Catalyzed by Ruthenium Bis(arylimino)acenaphthene Complexes. Chemcatchem 2010, 2, 1150-1164.

(23) Mondal, P.; Agarwala, H.; Jana, R. D.; Plebst, S.; Grupp, A.; Ehret, F.; Mobin, S. M.; Kaim, W.; Lahiri, G. K. Sensitivity of a Strained C-C Single Bond to Charge Transfer: Redox Activity in Mononuclear and Dinuclear Ruthenium Complexes of Bis(arylimino)acenaphthene (BIAN) Ligands. Inorg Chem 2014, 53, 7389-7403.

(24) Ragaini, F.; Cenini, S.; Tollari, S.; Tummolillo, G.; Beltrami, R. Allylic amination of unactivated olefins by nitroarenes, catalyzed by ruthenium complexes. A reaction involving an intermolecular C-H functionalization. Organometallics 1999, 18, 928-942.

(25) Ragaini, F.; Cenini, S.; Borsani, E.; Dompe, M.; Gallo, E.; Moret, M. Synthesis of Narylpyrroles, hetero-Diels-Alder adducts, and allylic amines by reaction of unfunctionalized dienes with nitroarenes and carbon monoxide, catalyzed by $\mathrm{Ru}(\mathrm{CO})(3)(\mathrm{Ar}-\mathrm{BIAN})$. Organometallics 2001, 20, 33903398. 
(26) Merino, E.; Poli, E.; Diaz, U.; Brunel, D. Synthesis and characterization of new ruthenium $\mathrm{N}$-heterocyclic carbene Hoveyda II-type complexes. Study of reactivity in ring closing metathesis reactions. Dalton Trans 2012, 41, 10913-10918.

(27) Al-Hashimi, M.; Tuba, R.; Bazzi, H. S.; Grubbs, R. H. Synthesis of Polypentenamer and Poly(Vinyl Alcohol) with a Phase-Separable Polyisobutylene-Supported Second-Generation HoveydaGrubbs Catalyst. Chemcatchem 2016, 8, 228-233.

(28) Gottumukkala, A. L.; Teichert, J. F.; Heijnen, D.; Eisink, N.; van Dijk, S.; Ferrer, C.; van den Hoogenband, A.; Minnaard, A. J. Pd-Diimine: A Highly Selective Catalyst System for the Base-Free Oxidative Heck Reaction. J Org Chem 2011, 76, 3498-3501.

(29) Al-Hashimi, M.; Hongfa, C.; George, B.; Bazzi, H. S.; Bergbreiter, D. E. A phase-separable second-generation hoveyda-grubbs catalyst for ring-opening metathesis polymerization ( $\mathrm{vol} 50, \mathrm{pg}$ 3954, 2012). J Polym Sci Pol Chem 2012, 50, 5211-5212.

(30) Al-Hashimi, M.; Hongfa, C.; George, B.; Bazzi, H. S.; Bergbreiter, D. E. A phase-separable second-generation hoveyda-grubbs catalyst for ring-opening metathesis polymerization. J Polym Sci Pol Chem 2012, 50, 3954-3959.

(31) Bantreil, X.; Sidi-Ykhlef, M.; Aringhieri, L.; Colacino, E.; Martinez, J.; Lamaty, F. Comprehensive study on olefin metathesis in PEG as an alternative solvent under microwave irradiation. J Catal 2012, 294, 113-118.

\footnotetext{
'See, for example, (a) Lunsford, A. M.; Blank, J. H.; Moncho, S.; Haas, S. C.; Muhammad, S.; Brothers, E. N.; Darensbourg, M. Y.; Bengali, A. A. "Catalysis and Mechanism of H2 Release from Amine-Boranes by Diiron Complexes" Inorg. Chem. 2016, 55, 964. (b) Sohail, M.; Moncho, S.; Brothers, E.N.; Bengali, A. A. "Dehydrogenation of a Tertiary Amine-Borane by a Rhenium Complex" Chem. Comm., 2014, 50, 5874-5877. (c) Yempally, V.; Moncho, S.; Sohail, M.; Brothers, E.N.; Arndtsen B.A.; Bengali, A. A. "Oxidative Addition of Haloalkanes to Metal Centers: A Mechanistic Investigation" Organometallics, 2014, 33, 3591-3595.

ii This is already in your manuscript: Merino, E.; Poli, E.; Diaz, U.; Brunel, D. Synthesis and characterization of new ruthenium $\mathrm{N}$-heterocyclic carbene Hoveyda II-type complexes. Study of reactivity in ring closing metathesis reactions. Dalton Trans 2012, 41, 10913-10918.

iii Solans-Monfort, X; Pleixats, R.; Sodupe, M. Chem Eur J 201016 7331-7343

${ }^{i v}$ Núñez-Zarur, F.; Solans-Monfort, X; Pleixats, R.; Rodríguez-Santiago, L. Sodupe, M. Chem Eur J 201319 14553-14565

${ }^{\vee}$ Gaussian Development Version, Revision H.32, Frisch, M. J.; Trucks, G. W.; Schlegel, H. B.; Scuseria, G. E.; Robb, M. A.; Cheeseman, J. R.; Scalmani, G.; Barone, V.; Mennucci, B.; Petersson, G. A.; Nakatsuji, H.; Caricato, M.; Li, X.; Hratchian, H. P.; Izmaylov, A. F.; Bloino, J.; Zheng, G.; Sonnenberg, J. L.; Hada, M.; Ehara, M.; Toyota, K.; Fukuda, R.; Hasegawa, J.; Ishida, M.; Nakajima, T.; Honda, Y.; Kitao, O.; Nakai, H.; Vreven, T.; Montgomery, J. A., Jr.; Peralta, J. E.; Ogliaro, F.; Bearpark, M.; Heyd, J. J.; Brothers, E.; Kudin, K. N.; Staroverov, V. N.; Kobayashi, R.; Normand, J.; Raghavachari, K.; Rendell, A.; Burant, J. C.; Iyengar, S. S.; Tomasi, J.; Cossi, M.; Rega, N.; Millam, J. M.; Klene, M.; Knox, J. E.; Cross, J. B.; Bakken, V.; Adamo, C.; Jaramillo, J.; Gomperts, R.; Stratmann, R. E.; Yazyev, O.; Austin, A. J.; Cammi, R.; Pomelli, C.; Ochterski, J. W.; Martin, R. L.; Morokuma, K.; Zakrzewski, V. G.; Voth, G. A.; Salvador, P.; Dannenberg, J. J.; Dapprich, S.; Daniels, A. D.; Farkas, Ö.; Foresman, J. B.; Ortiz, J. V.; Cioslowski, J.; Fox, D. J., Gaussian, Inc., Wallingford CT, 2009.

${ }^{v i}$ Zhao, Y. ; Truhlar, D. G. A new local density functional for main-group thermochemistry, transition metal bonding, thermochemical kinetics, and noncovalent interactions. J. Chem. Phys., 2006, 125, 194101:1-18

vii Weigend, F.; Ahlrichs, R. Balanced basis sets of split valence, triple zeta valence and quadruple zeta valence quality for H to Rn: Design and assessment of accuracy Phys. Chem. Chem. Phys., 2005 , 7, 3297-3305.
} 
viii CYLview, 1.0b; Legault, C. Y., Université de Sherbrooke, 2009 (http://www.cylview.org). 